\title{
Disorder-induced mutation of quasi-normal modes in 1D open systems
}

\author{
Yury Bliokh, ${ }^{1,2}$ Valentin Freilikher, ${ }^{3,2}$ and Franco Nori ${ }^{2,4}$ \\ ${ }^{1}$ Department of Physics, Technion-Israel Institute of Technology, Haifa 32000, Israel, \\ ${ }^{2}$ CEMS, RIKEN, Wako-shi, Saitama 351-0198, Japan \\ ${ }^{3}$ Department of Physics, Jack and Pearl Resnick Institute, Bar-Ilan University, Israel \\ ${ }^{4}$ Department of Physics, University of Michigan, Ann Arbor, Michigan 48109-1040, USA
}

\begin{abstract}
We study the relation between quasi-normal modes (QNMs) and transmission resonances (TRs) in one-dimensional (1D) disordered systems. We show for the first time that while each maximum in the transmission coefficient is always related to a QNM, the reverse statement is not necessarily correct. There exists an intermediate state, where only part of the QNMs are localized and these QNMs provide a resonant transmission. The rest of the solutions of the eigenvalue problem (denoted as strange quasi-modes) are never found in regular open cavities and resonators, and arise exclusively due to random scatterings. Although these strange QNMs belong to a discrete spectrum, they are not localized and not associated with any anomalies in the transmission. The ratio of the number of the normal QNMs to the total number of QNMs is independent of the type of disorder, and deviates only slightly from the constant $\sqrt{2 / 5}$ in rather wide ranges of the strength of a single scattering and the length of the random sample.
\end{abstract}

Wave processes in open systems can be described in terms of quasi-normal modes (QNMs), which are a generalization of the notion of normal modes for closed systems, to open structures, [1 9]. The corresponding eigenfrequencies are complex, so that the imaginary parts characterize the lifetime of the quasi-normal states. Regarding the transmission of radiation through random media, it is more appropriate to use an alternative approach based on transmission resonances (TR): open channels, through which the radiation transmits with high efficiency [3, 10 22].

Recently, physicists came to realize that focusing radiation into such channels could not only enhance the total intensity transmitted through strongly-scattering media, but also: significantly improve images blurred by random scattering, facilitate the detection and location of objects, provide optical tomography at very large depths, etc. [13, 17, 18, 20, 23]. To efficiently excite transmission resonances, it is preferable to treat them as superpositions of QNMs, with which the incident signal can be coupled by a properly-shaped wavefront [13, 14]. The great potential of such algorithms for a host of practical applications is obvious. This is why the relation between transmission resonances and QNMs have recently attracted particular attention of both the physical [12, 24 28] and mathematical communities [1].

It is now universally accepted that in open systems (e.g., quantum potential wells, optical cavities, or microwave resonators) each maximum in the transmission coefficient (i.e., transmission resonance) is associated with a QNM, so that the resonant frequency is close to the real part of the corresponding eigenvalue. QNMs and TRs are often considered identical. For example, the solutions of the eigenvalue problem (with no incoming waves), which in physics are unambiguously called QNMs, in the mathematical community dealing with the scattering inverse problem, are termed transmission eigenvalues [1]. However, the connection between QNMs and TRs is not that simple and, despite extensive research and much recent progress, still needs a better physical understanding and mathematical justification, at least for disordered systems.

To this end, it is instructive to look for insights the 1D limit because its spectral and transport properties are better understood. It is well-known [29] that the transmission of a long enough 1D disordered system is typically (for most of the frequencies) exponentially small. At the same time, there exists a set of frequencies where the transmission coefficient has local maxima (resonances in transmission), some of them close to one [30]. Each resonance is a transmission channel and is always associated with a QNM determined in a standard way as a solution with outgoing boundary conditions. The reverse statement, that each QNM manifests itself as a transmission resonance, although never has been questioned, is usually taken as obvious and self-evident, perhaps because it is always the case in all regular (homogeneous or periodic) quantum-mechanical and optical open structures.

Here we show, both numerically and analytically, that in 1D disordered systems there exist two types of QNMs: ordinary QNMs, that provide resonance transmission peaks, and "strange" QNMs unrelated to any anomalies in the transmission spectrum. These strange modes exist exclusively due to random scatterings and arise already in the ballistic regime with weak disorder. Although they belong to the discrete spectrum, their eigenfunctions are not localized. The imaginary parts of the strange QNMs eigenfrequencies vary with increasing disorder in a highly unusual manner. Indeed, typically, the stronger the disorder is, the more confined the system becomes, which implies that the eigenfrequencies should approach the real axis. However, the imaginary part of a strange mode's eigenfrequency either increases from the onset of disorder, or goes down anomalously slowly. Most 
surprisingly, up to rather strong disorder, the average ratio of the density (in the frequency domain) of strange modes to the total density of QNMs, being independent of the type of disorder, remains close to the constant $\sqrt{2 / 5}$ in wide ranges of the strength of disorder and of the total length of the system. The value $\sqrt{2 / 5}$ follows from the general statistical properties of random trigonometric polynomials [31]. As the disorder keeps growing, eventually all strange quasimodes turn normal. Therefore these results can be interpreted as a manifestation of the existence (in 1D random systems, at least) of an intermediate regime, at which in any finite-frequency interval, only a part of the quasimodes are localized and provide resonant transmission.

We consider a generic 1D system composed of $N+1$ scatterers separated by $N$ intervals and attached to two semi-infinite leads. Two problems are associated with such systems. The first one is finding solutions $\psi(x, t)$ of the wave equation satisfying the outgoing boundary conditions, which means that there are no right/leftpropagating waves in the left/right lead. The eigenfunction solution $\psi_{n}(x, t)$ of this problem is the superposition of two counter-propagating monochromatic waves $\psi_{n}(x)^{( \pm)} e^{-i \omega_{n} t}$. In any $j$ th layer $\psi_{n}^{( \pm)}(x)=\psi_{n, j}^{( \pm)}(x)=$ $a_{n, j}^{( \pm)} e^{ \pm i k_{n} x}$. The amplitudes $a_{n, j}^{( \pm)}$in adjacent layers are connected by a transfer matrix. The wave numbers $k_{n}$ are complex-valued and form the discrete set (poles of the scattering matrix) $k_{n}^{(\bmod )}=k_{n}^{\prime}-i k_{n}^{\prime \prime}, k^{\prime \prime}>0$, and frequencies $\omega_{n}^{(\bmod )}=c k_{n}$. The corresponding eigenfunctions are the so-called QNMs. Note that all distances hereafter are measured in optical lengths. The second problem is the transmission of an incident wave through the system. The set of wave numbers and corresponding fields inside the system for which the transmission coefficient reaches its local maximum are the so-called TRs. Evidently these two problems are interrelated. In particular, the density of QNSs at a frequency $\omega$ is proportional to the derivative with respect to frequency of the phase of the complex transmission coefficient [32, 33].

The goal of this paper is to establish the relation between the spectra and wave functions of QNMs and TRs.

In what follows, the scatterers and the distances between them are characterized by the reflection coefficients $r_{i} \equiv r_{0}+\delta r_{i}$ and lengths $d_{i} \equiv d_{0}+\delta d_{i}$, respectively. The random values $\delta r_{i}$ and $\delta d_{i}$ are distributed in certain intervals, and $\left\langle\delta r_{i}\right\rangle=0$ and $\left\langle\delta d_{i}\right\rangle=0$. Here, $\langle\ldots\rangle$ stands for the value averaged over the sample. The last condition means that the total length $L$ of the system is equal to $N d_{0}$ and therefore any random realization with the same $N$ contains the same number of QNMs.

To explicitly introduce the tunable strength $s$ of disorder, we replace all reflection coefficients, except for those at the left, $r_{L}$, and right, $r_{R}$, edges of the system by $s r_{i}$, and assume (unless otherwise specified) that the coefficients $r_{i}$ are homogeneously distributed in the interval
$(-1,1)$. This notation enables keeping track of the evolution of the QNM eigenvalues $k_{n}^{(\bmod )}$ and of the resonant wave vectors $k^{(\mathrm{res})}$ when the disorder increases from zero $(s=0)$ while the reflection coefficients $r_{L}$ and $r_{R}$ at the semitransparent boundaries remain constant.

When $s=0$, (i.e., no disorder) the real and imaginary parts of the QNM eigenvalues $k_{n}^{(\bmod )}$ are

$$
\begin{aligned}
& k_{n}^{\prime}=\frac{1}{2 L} \cdot\left\{\begin{array}{r}
\pi+2 \pi n, \\
2 \pi n,
\end{array} \quad \text { when } r_{L} r_{R}>0,\right. \\
& k_{n}^{\prime \prime}=-\frac{1}{2 L} \ln \left|r_{L} r_{R}\right| .
\end{aligned}
$$

The wave intensity, defined as $I_{n, j}=\left|\psi_{n, j}^{(+)}\right|^{2}+\left|\psi_{n, j}^{(-)}\right|^{2}$ is distributed along the system as $I_{n}\left(x_{j}\right) \propto \cosh \left[2 k^{\prime \prime}\left(x_{j}-\right.\right.$ $\left.\left.x^{*}\right)\right]$, where $x^{*}=L\left[1-\ln \left(\left|r_{R} / r_{L}\right|\right) / \ln \left(\left|r_{R} r_{L}\right|\right)\right] / 2$. When $\left|r_{L}\right|=\left|r_{R}\right|$, the minimum of the intensity is located at the centre of the system, and in an asymmetric case shifts to the boundary with a higher reflection coefficient. This property will be used when analyzing the behavior of the QNMs when the disorder parameter $s$ grows.

It is easy to show that when $s=0$ the wave numbers $k_{n}^{(\text {res })}$ of the transmission resonances coincide with the real parts $k_{n}^{\prime}$ given by Eq. (11). Thus, in the homogeneous resonator, there is a one-to-one correspondence between QNMs and TRs. The same correlation exists also in periodic systems (periodic sets $r_{i}$ and $d_{i}$ ) [34].

The question now is whether this relationship survives in the disordered system, when $s \neq 0$. There is strong evidence [3, 11 13, 15] that for every resonance there is a corresponding QNM. However, as we show below, the reverse statement is not valid: there are certain QNMs which cannot be associated with any resonance.

Figure 1 1 shows the evolution of the eigennumbers $k_{n}^{\bmod }$ in the complex plane $\left(k^{\prime}, k^{\prime \prime}\right)$ as $s$ grows. Initially, when $s=0$, all eigenumbers are equidistantly located on the line $k^{\prime \prime}=$ const, in agreement with Eqs. (1), 2). As soon as disorder arises $(s \neq 0)$ and increases, the eigenvalues separate into two essentially different types. Indeed, with $s$ increasing, the points \#1-3,5,7,8,10,12,13 in Fig. 1 move towards the real axis ( $k_{i}^{\prime \prime}$ decrease) with approximately the same "velocity" (ordinary QNMs). The rest of the points (strange QNMs) either shift down substantially more slowly $(\# 0,6,9)$ or, even more surprisingly, move away from the real axis (points 4 and 11). The latest modes are highly unusual because disorder makes them more leaky. This is quite the opposite to the hitherto observed and well understood increase of the lifetime of the eigenstates due to multiple scattering.

The difference between the ordinary and strange QNMs goes beyond the evolution of the eigenvalues and manifests itself also in the the spatial distribution of the QNM intensity inside the system. As an example, the spatial distributions along the system of the intensities $I_{5, j}$ and $I_{6, j}$ of QNMs \#5 and 6 are presented in Fig 2. for different values of $s$. Note that the difference be- 


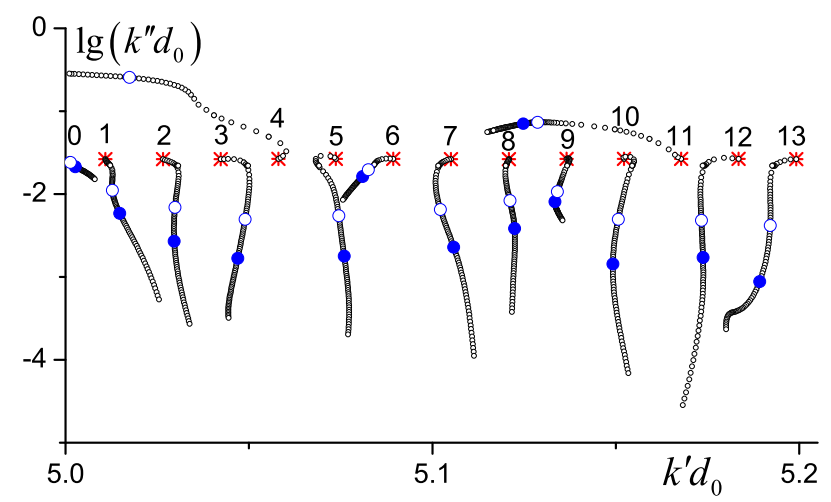

FIG. 1. (color online) Motion of the QNMs eigenvalues, $k_{n}^{\text {(mod) }}=k_{n}^{\prime}-i k_{n}^{\prime \prime}$, as the degree $s$ of disorder grows. Red asterisks mark the initial positions with no disorder $(s=0)$. Red open circles and blue solid circles show the positions of the QNMs eigenvalues at $s=0.1$ and $s=0.2$, respectively. Note that ordinary QNMs shift down when increasing disorder while some strange QNMs (e.g. \#4 and 11) shift up.

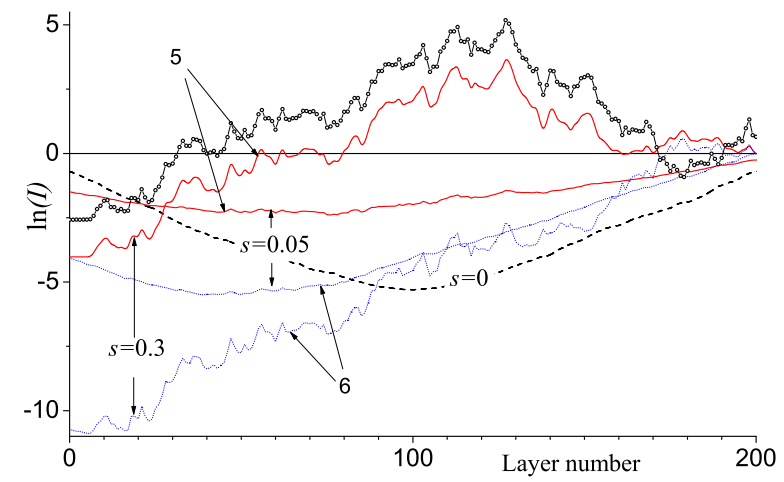

FIG. 2. (color online) Spatial distribution of the intensity $I(j)$ of QNMs \#5 (solid curves) and \#6 (dotted curves) for different values of the disorder strength $s$. The dashed black curve corresponds to a homogeneous resonator $\left(s=0, r_{L}=\right.$ $\left.-r_{R}=0.005\right)$. The resonance intensity distribution for $s=$ 0.3 is shown by circles.

tween the imaginary parts $k^{\prime \prime}$ of the eigenvectors 5 and 6 increases as $s$ increases (see Fig. 1). Despite the fact that the initial $(s=0)$ distributions are identical, even small disorder $(s=0.05)$ deforms the distributions $I_{5, j}$ and $I_{6, j}$ in very different ways. The distribution $\left.I_{5, j}\right|_{s=0.05}$ is similar to $\left.I_{5, j}\right|_{s=0}$, but has a much less pronounced minimum. By analogy with a homogeneous resonator, this can be interpreted as the growth of the effective reflection coefficients $r_{L}$ and $r_{R}$, which agrees well with the statement that the wave lifetime increases when disorder becomes stronger. For larger $s, I_{5, j}$ tends to manifest the behaviour typical for QNM in the localized regime. In contrast, the intensity evolution of QNM \#6 is similar to that in the homogeneous resonator, whose right wall becomes more transparent. QNMs \#4 and 11 also demonstrate the same behaviour, but the effective transparency of one of the "walls" increases much faster when the degree of disorder $s$ grows.

We also consider the propagation of a monochromatic wave through the same system. When $s=0$, the number of resonances $N_{\text {res }}$ is equal to the number of QNMs, $N_{\text {mod }}$, and all $k_{n}^{(\mathrm{res})}$ coincide with the real parts $k_{n}^{\prime}$ of QNMs. When disorder is introduced, $s \neq 0$, each $k_{n}^{\text {(res) }}$ remains close to the $k_{n}^{\prime}$ of the corresponding ordinary QNM: $k_{n}^{(\text {res })}(s) \simeq k_{n}^{\prime}(s)$. The spatial intensity distributions of QNM \#5 and of the corresponding TR are also similar, up to small details (see Fig. 2).

However, the transmission resonances whose frequencies at $s=0$ are equal to the real parts of the eigenvalues of the strange QNMs, disappear when the mean value of the reflection coefficients $s\left\langle r_{i}\right\rangle$ becomes of the order of $r_{L, R}$. Figure 3 demonstrates this behavior. Here, the role of the reflection coefficients $r_{R, L}\left(r_{R}=r_{L}=0.005\right.$ in Figs. 2 and 31) is only to specify the TRs and QNMs at $s=0$.

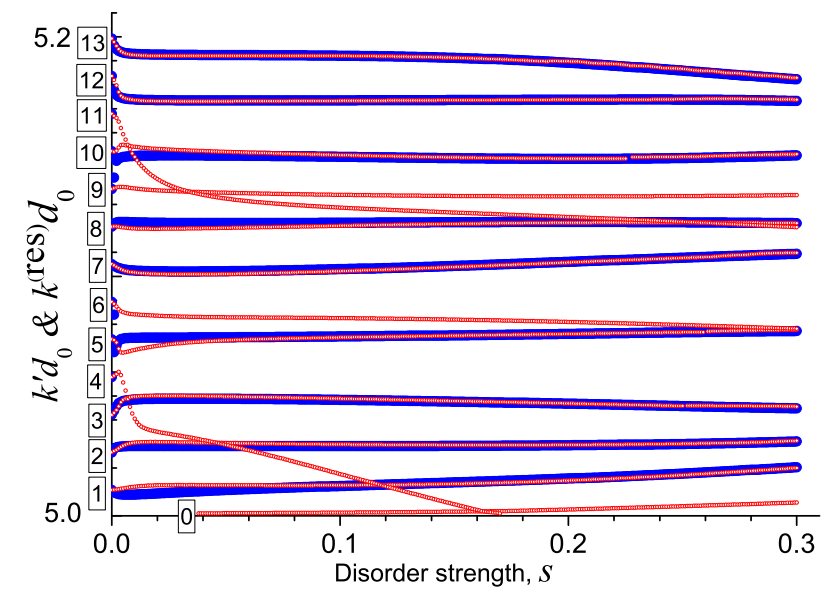

FIG. 3. (color online) Dependencies $k_{\text {res }}(s)$ (thick blue solid circles) and $k^{\prime}(s)$ (thin open red circles). QNMs are numbered as in Fig. 22 It is seen that for ordinary QNMs, $k^{\text {res }}(s)$ and $k^{\prime}(s)$ practically coincide, whereas there are no resonances associated with strange QNMs (\#0,4,6,9,11).

Thus, any TR has its partner among QNMs, but the reverse is not true: there are strange QNMs that are not associated with any maxima in the transmission, as it is shown in Fig. 4, and therefore do not have co-partners between resonances. In other words, in a given wave number interval $\Delta k$, the statistically-averaged number of TRs, $N_{\text {res }}$, is smaller than the statistically-averaged number of QNMs, $N_{\text {mod }}=\Delta k L / \pi$, and does not depend on the degree of disorder. This fact was noticed in the numerical calculations in 28].

Surprisingly, when $s \rightarrow 0$, the ratio $N_{\text {res }} / N_{\text {mod }}$ is a universal constant $\sqrt{2 / 5}$, independent of the type of disorder, and remains practically independent on the degree of disorder and the length $L$ of the system in a rather 


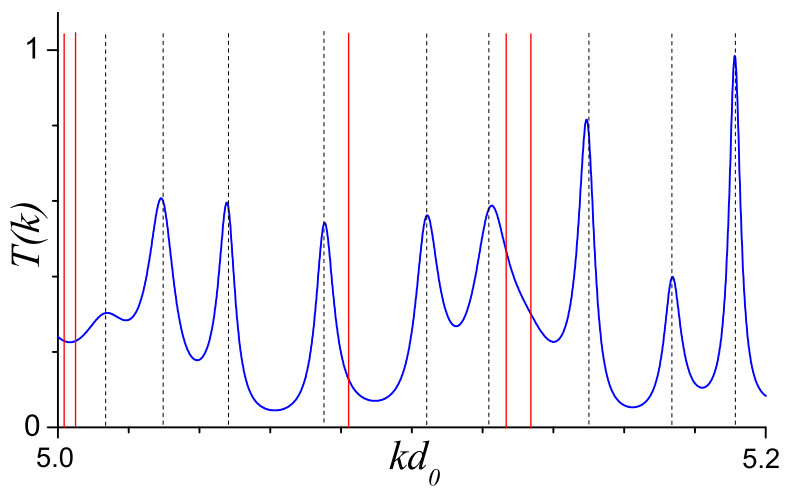

FIG. 4. (color online) Transmission spectrum $T(k)$ at $s=$ 0.15 . The black dashed (red solid) vertical lines indicate the $k_{n}^{\prime}$ values of ordinary (strange) QNMs.

broad range of these parameters.

Figure 5 shows the ratio $N_{\text {res }} / N_{\text {mod }}$ as a function of $s$, statistically averaged over $10^{4}$ random realizations and normalized by $\sqrt{2 / 5}$, for various lengths $L$ in the case $r_{L, R}=0$. It is important to note that the localiza-

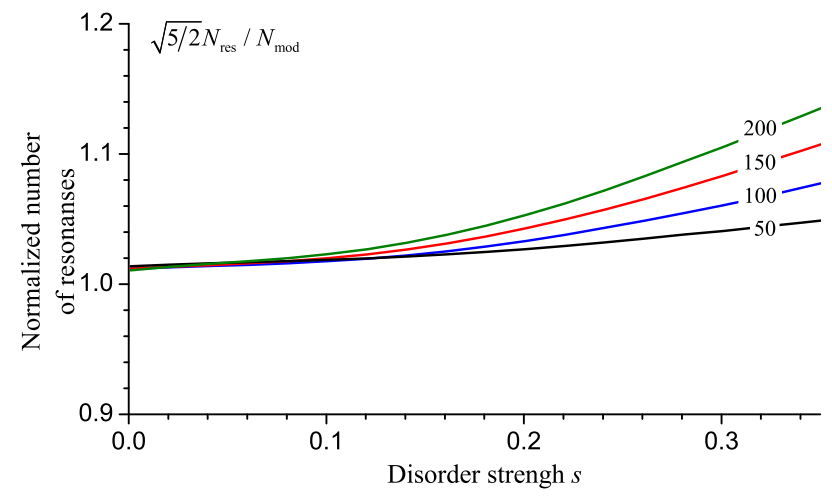

FIG. 5. (color online) Normalized ratio $\sqrt{5 / 2} N_{\text {res }} / N_{\text {mod }}$ versus the degree of disorder $s$ for systems of various lengths $L=N d_{0}$ ( $N$ is the number of layers).

tion length (measured in numbers of layers) $N_{\text {loc }} \propto s^{-2}$, and this is less than 20 for $s=0.3$. This means that $N_{\text {res }} / N_{\text {mod }} \simeq \sqrt{2 / 5}$ even when the system dimension exceeds considerably the localization length.

Figure 3 shows that the difference betweens $N_{\text {res }}$ and $N_{\text {mod }}$ appears when $s$ is very small so that $N_{\text {loc }} \gg N$, and remains practically unchanged even when $s$ is rather large so that $N_{\text {loc }} \ll N$. This means that the origin of this phenomenon is not specifically related to localization and can be studied when $s$ is arbitrarily small.

To calculate the average number of TRs in the limit $s \ll 1$, we use the single-scattering approximation and write the total reflection coefficient $r(k)$ of the whole system as:

$$
r(k)=\Sigma_{n=1}^{N} r_{n} e^{2 i k x_{n}},
$$

where $x_{n}$ is the coordinate of the $n$-th scatterer. The values $k_{\max }$, where the transmission coefficient, $T(k)=$ $1-|r(k)|^{2}$, has local maxima, are defined as the zeros of the function $f(k) \equiv d|r(k)|^{2} / d k=2 \operatorname{Re}\left[r(k) d r^{*}(k) / d k\right]$ :

$$
f\left(k_{\max }\right)=4 \operatorname{Im} \Sigma_{n=1}^{N} \sum_{m=1}^{N} r_{n} r_{m} x_{m} e^{2 i k_{\max }\left(x_{n}-x_{m}\right)}=0 .
$$

Assuming first that $\delta d_{i}=0$, then $f(k)$ becomes

$$
\begin{array}{r}
f(k) \propto \Sigma_{n=1}^{N} \Sigma_{m=1}^{N} r_{n} r_{m}(m-n) \sin \left[2 k(m-n) d_{0}\right] \\
=\Sigma_{l=1}^{N} \sin \left(2 k l d_{0}\right)\left\{\Sigma_{n=1}^{N-l} r_{n+l} r_{n} l\right. \\
\left.+\sum_{n=l}^{N} r_{n-l} r_{n} l\right\} \equiv \Sigma_{l=1}^{N} \sin \left(2 k l d_{0}\right) a_{l} .
\end{array}
$$

Eq. (5) is the trigonometric sum $\sum_{l=1}^{N} a_{l} \sin \left(\nu_{l} k\right)$ with "frequencies" $\nu_{l}=2 l d_{0}$ and random coefficients $a_{l}$. The statistics of the zeroes of random polynomials have been studied in 31, where it is shown that the statisticallyaveraged number of real roots $N_{\text {root }}$ of the sum of this type at a certain interval $\Delta k$ is

$$
N_{\text {root }}=\frac{\Delta k}{\pi} \sqrt{\frac{\Sigma \nu_{l}^{2} \sigma_{l}^{2}}{\Sigma \sigma_{l}^{2}}},
$$

where $\sigma_{l}^{2}=\operatorname{Var}\left(a_{l}\right)$ is the variance of the coefficients $a_{L}=\sum_{n=1}^{N-l} r_{n+l} r_{n} l+\sum_{n=l}^{N} r_{n-l} r_{n} l$. When $N \gg 1$,

$$
\operatorname{Var}\left(a_{l}\right) \simeq 2(N-l) l^{2} \sigma_{0}^{4},
$$

where $\sigma_{0}^{2}=\operatorname{Var}(r)$. The sums in Eq. (6) can be calculated using Eq. (7), which yields [35]:

$$
\begin{aligned}
\Sigma_{l=1}^{N} \sigma_{l}^{2} & =2 \sigma_{0}^{4} \Sigma_{l=1}^{N} l^{2}(N-l) \simeq \frac{1}{6} \sigma_{0}^{4} N^{4}, \\
\Sigma_{l=1}^{N} \nu_{l}^{2} \sigma_{l}^{2} & =8 d_{0}^{2} \Sigma_{l=1}^{N} \sigma_{0}^{4} l^{4}(N-l) \simeq \frac{4}{15} d_{0}^{2} N^{6} \sigma_{0}^{4} .
\end{aligned}
$$

From Eqs. (6) and (8) we obtain

$$
N_{\text {root }}=\frac{2 \Delta k N d_{0}}{\pi} \sqrt{\frac{2}{5}}=2 \frac{\Delta k L}{\pi} \sqrt{\frac{2}{5}},
$$

where $L=N d_{0}$. Since the number of minima of the reflection coefficient is equal to the number of TRs, $N_{\text {res }}=N_{\text {root }} / 2$, and the number $N_{\text {mod }}$ of QNMs in the same interval $\Delta k$ is $N_{\text {res }}=\Delta k L / \pi$, from Eq. 9 it follows that

$$
N_{\text {res }} / N_{\text {mod }}=\sqrt{2 / 5}
$$

This analytically-calculated relation agrees perfectly with the results of numerical calculations performed without assuming any periodicity of the scatterers. To calculate this ratio for more general situations, when the distances between the scatterers are also random $\left(\delta d_{i} \neq 0\right)$, the frequencies $\nu=2 l d_{d}$ in Eq. (5) should be replaced by $\nu=2\left|x_{m}-x_{m \pm l}\right|$. Since the main contribution to the sums in Eq. (6) is given by the terms with large $l \sim N$, the mean value of $\left|x_{m}-x_{m \pm l}\right|$ can be replaced by $l d_{0}$, in the case of a homogeneous distribution of the distances 
$d_{n}$ along the system. This ultimately leads to the same result Eq. (101).

In summary, it is well known that there is a one-toone correspondence between the QNMs of a regular open system (wave resonator or quantum cavity) and its transmission resonances: each QNM is unambiguously associated with a TR, and vice versa. In this paper, we show for the first time that in 1D random structures, this reciprocity is broken: any weak disorder mutates part of the eigenstates so that the corresponding resonances in the transmission disappear and the density of TR becomes smaller than the total density of states. Although the strange modes belong to a discrete spectrum, the spatial structure of the eigenfunctions differs drastically from that of the ordinary states and show no sign of localization. It is significant that while the strange modes do not show up in the amplitude of the transmission coefficient, in the phase of the transmitted field they manifest themselves in just the same way as the ordinary modes do. Indeed, the numerical calculations show that the derivative of this phase with respect to the frequency gives the total density of QNMs, which includes both the normal ordinary and strange ones. When the disorder is weak (but strong enough to localize the ordinary modes), the ratio of the number of TRs to the total number of QNMs in a frequency interval $\Delta \omega \rightarrow \infty$ is independent of the type of disorder and anomalously weakly deviates from a universal constant, $\sqrt{2 / 5}$, when the strength of disorder and the length of the random sample increase. This constant coincides with the one analytically calculated in the weak single-scattering approximation ensemble-averaged ratio $N_{\text {res }} / N_{\text {mod }}$. If the strength $s$ of disorder grows, ultimately all strange quasimodes become ordinary. This means that in 1D random systems there exists an intermediate, so far unknown regime, at which in any finitefrequency interval, only a part of the quasimodes are localized and provide resonant transmission.

We gratefully acknowledge stimulating discussion with K. Bliokh. We specially thank M. Dennis who drew our attention to the paper [31].

This research is partially supported by the RIKEN iTHES Project, MURI Center for Dynamic MagnetoOptics, and a Grant-in-Aid for Scientific Research (S).

[1] F. Cakoni, H. Haddar (Editors), Inverse Problems 29, Topical Issue, (2013).

[2] E.S.C. Ching, P.T. Leung, A. Maassen van den Brink, W.M. Suen, S.S. Tong, and K. Young, Rev. Mod. Phys. 70, 1545 (1998).

[3] J. Wang and A. Genack, Nature 471, 345 (2011).

[4] N.Hatano and G. Ordonez, arXiv:1405.6683 (2014).

[5] Wonjun Choi, Q-Han Park, and Wonshik Choi, Opt. Exp. 20, 20721 (2012).

[6] C. Sauvan, J.P. Hugonin, I.S. Maksymov, and P. Lalanne,
arXiv: 1304.8110

[7] C. Vanneste and P. Sebbah, Phys. Rev. A 79, 041802 (2009).

[8] F.A. Pinheiro, M. Rusek, A. Orlowski, and B. van Tiggelen, Phys. Rev. E 69, 026605 (2004).

[9] P. Sebbah, B. Hu, J. Klosner, and A. Genack, Phys. Rev. Lett. 96, 183902 (2006).

[10] O. Dorokhov, Solid State Com. 44, 915 (1982); ibid. 51, 381, (1984).

[11] Wonjun Choi, A. Mosk, Q-Han Park, and Wonshik Choi, Phys. Rev. B 83, 134207 (2011).

[12] A. Pena, A. Girschik, F. Libisch, S. Rotter, and A. Chabanov, Nature Communications 5, 1 (2014).

[13] S. Liew, S. Popoff, H. Cao, A. Mosk, and W. Vos, arXiv:1401.5805 (2014).

[14] S.M. Popoff, A. Goetschy, S.F. Liew, A.D. Stone, and H. Cao, Phys. Rev. Lett. 112, 133903 (2014).

[15] X. Cheng, C. Tian, and A. Genack, Phys. Rev. B 88, 094202 (2013).

[16] Z. Shi and A.Z. Genack, Phys. Rev. Lett. 108, 043901 (2012).

[17] I. Vellekoop and A. Mosk, Phys. Rev. Lett. 101, 120601 (2008).

[18] Z. Shi, M. Davy, J. Wang, and A. Genack, Opt. Lett. 38, 2714 (2013).

[19] B. Gérardin, J. Laurent, A. Derode, C. Prada, and A. Aubry, arXiv:1404.2092 (2014).

[20] A. Mosk, A. Lagendijk, G. Lerosey, and M. Fink, Nature Phot. 6, 283 (2012).

[21] K. Bliokh, Y. Bliokh, V. Freilikher, S. Savel'ev, and Franko Nori, Rev. Mod. Phys. 80, 1201 (2008).

[22] K. Bliokh, Y. Bliokh, V. Freilikher, and Franko Nori, "Anderson Localization of Light in Layered Dielectric Structures", in Optical properties of photonic structures: interplay of order and disorder, ed. by. M. Limonov and R. De La Rue, (CRC Press) p.57 - 86 (2012).

[23] A. Goetschy and A. Stone, Phys. Rev. Lett. 111, 063901 (2013).

[24] W. L. Vos, T. W. Tukker, A. P. Mosk, A. Lagendijk, and W. L. IJzerman, Appl. Opt. 52, 2602 (2013).

[25] M. Kim, W. Choi, C. Yoon, G.H. Kim, and W. Choi, Opt. Lett. 38, 2994 (2013).

[26] Wonjun Choi, M. Kim, D. Kim, C. Yoon, C. Fang-Yen, Q. Park, and Wonshik Choi, arXiv:1308.6558 (2013).

[27] L. Chen, C. Lv, and X. Jiang, Comp. Phys. Commun. 183, 2513 (2012).

[28] Y. Bliokh, E. Chaikina, N. Lizárraga, E. Mendez, V. Freilikher, and Franco Nori, Phys. Rev B 86, 054204 (2012).

[29] I. Lifshitz, S. Gredeskul, L. Pastur, Introduction to the Theory of Disordered Systems (Wiley, New York, 1989).

[30] K. Yu. Bliokh, Yu. P. Bliokh, and V. D. Freilikher, J. Opt. Soc. of America B 21, 113 (2004); K. Yu. Bliokh, Yu. P. Bliokh, V. Freilikher, A. Z. Genack, B. Hu, and P. Sebbah, Phys. Rev. Lett. 97, 243904 (2006); K.Y. Bliokh, Y.P. Bliokh, V. Freilikher, A.Z. Genack, and P. Sebbah, Phys. Rev. Lett. 101, 133901 (2008); I.V. Shadrivov, K.Y. Bliokh, Y.S. Kivshar, Y.P. Bliokh, and V. Freilikher, Phys. Rev. Lett. 104, 123902 (2010); K.Y. Bliokh, S.A. Gredeskul, P. Rajan, I.V. Shadrivov, and Y.S. Kivshar, Phys. Rev. B 85, 014205 (2012).

[31] A. Edelman and E. Kostlan, Bull. Am. Math. Soc. 32, No. 1, 1-37 (1995), p.14.

[32] A.Y. Avishai and Y. Band, Phys. Rev. B 32, 2674 (1985).

[33] B.E. Akkermans, G. Dunne, and E. Levy, 
arXiv:1210.7409v2 (2012).

[34] A. Settimi, S. Severini, N. Mattiucci, C. Sibilia, M. Centini, G. D'Aguanno, and M. Bertolotti, Phys. Rev. E 68,
026614 (2003)

[35] I.S. Gradshteyn and I.M. Ryzhik, Table of Integrals, Series, and Products, Seventh Edition, Academic Press, 2007, pp.1,2 